PROCEEDINGS OF THE

AMERICAN MATHEMATICAL SOCIETY

Volume 131, Number 5, Pages 1583-1590

S 0002-9939(02)06973-3

Article electronically published on December 16, 2002

\title{
AN INVERSE PROBLEM FOR AN INHOMOGENEOUS CONFORMAL KILLING FIELD EQUATION
}

\author{
ZIQI SUN
}

(Communicated by David S. Tartakoff)

\begin{abstract}
Let $g$ be a $C^{2, \alpha}$ Riemannian metric defined on a bounded domain $\Omega \subset R^{2}$ with $C^{3, \alpha}$ boundary and let $X$ be a $C^{2, \alpha}$ vector field on $\bar{\Omega}$ satisfying $\left.X\right|_{\partial \Omega}=0$. We show that if $l$ is a gradient field of a solution $u$ to the equation $\triangle_{g} u-\left\langle\nabla_{g} \sigma, \nabla_{g} u\right\rangle_{g}=0$ on $\Omega$, then both inner products $\langle l, X\rangle_{g}$ and $\left\langle l^{\perp}, X\right\rangle_{g}$ are uniquely determined by the restriction of the tensor $\mathcal{L}_{X}(g)-\left(e^{\sigma} \nabla_{g}\right.$. $\left.\left(e^{-\sigma} X\right)\right) g$ to the gradient field $l$, where $\mathcal{L}_{X}(g)$ is the Lie derivative of the metric tensor $g$ under the vector field $X$ and $\sigma=\log \sqrt{\operatorname{det}(g)}$. This work solves a problem related to an inverse boundary value problem for nonlinear elliptic equations.
\end{abstract}

\section{INTRODUCTION}

The goal of this paper is to present a solution to an inverse problem for the inhomogeneous conformal Killing field equation. The inverse problem originates from a study of the inverse boundary value problems for a class of quasilinear elliptic equations initiated in [H-Su]. The inverse boundary value problems for semilinear and quasilinear elliptic equations have been studied extensively in the last few years [I 1], [I 2], [I-N], I-S], [Su], [Su-U]. It is well-known that an inverse boundary value problem for a quasilinear elliptic equation can be reduced to an inverse boundary value problem for the corresponding linearized elliptic equation through a linearization procedure I 1. This linearization procedure solves the quasilinear problem almost immediately when the equation is an isotropic one [Su]. However, when one deals with the quasilinear anisotropic elliptic equation, the linearization procedure reduces the original quasilinear problem to a family of linearized problems depending on the boundary values, and another argument is thus needed to show that the diffeomorphism obtained from the linearization is actually independent of the boundary values. If the original quasilinear anisotropic problem involves merely the unknown solution in its quasilinear coefficient, one can use a second linearization procedure to achieve the above goal since the required linearization is performed only at the constant boundary values $[\mathrm{Su}$, $[\mathrm{Su}-\mathrm{U}]$. In the case where one has a quasilinear anisotropic problem involving the gradient of the unknown solution, the above second linearization argument fails to work since in this case one needs to linearize the equation at a general boundary value. The recent work of [H-Su] has been devoted to solve this problem, in which the second linearization

Received by the editors January 8, 2002

2000 Mathematics Subject Classification. Primary 35R30, 53C21.

(C)2002 American Mathematical Society 
has been replaced by a more subtle geometric analysis that reduces the problem to an inverse problem for the inhomogeneous conformal Killing field equation. This paper is devoted to solve this problem. We refer the readers to [U] for a general discussion on the field of inverse boundary value problems.

Let $\Omega \subset R^{2}$ be a bounded domain with $C^{3, \alpha}$ boundary. Let $g$ be a $C^{2, \alpha}$ Riemannian metric defined on $\bar{\Omega}$ and let $X$ be a $C^{2, \alpha}$ vector field on $\bar{\Omega}$. The conformal Killing field equation considered in this paper is concerned with the tensor

$$
\mathcal{L}_{X}(g)-\left(e^{\sigma} \nabla_{g} \cdot\left(e^{-\sigma} X\right)\right) g
$$

where $\mathcal{L}_{X}(g)$ is the Lie derivative of the metric tensor $g$ under the vector field $X$ and $\sigma=\log \sqrt{\operatorname{det}(g)}$. Here we use $\nabla_{g}$. to denote the divergent operator under the metric $g$. In this paper, we are mainly interested in the case when $X$ satisfies $\left.X\right|_{\partial \Omega}=0$.

Let $l$ be the $\left(C^{2, \alpha}\right)$ gradient field of a $C^{3, \alpha}$ solution $u$ to the equation

$$
\triangle_{g} u-\left\langle\nabla_{g} \sigma, \nabla_{g} u\right\rangle_{g}=0
$$

on $\bar{\Omega}$. Then $l$ satisfies the equation

$$
\nabla_{g} \cdot l-\left\langle\nabla_{g} \sigma, l\right\rangle_{g}=0 .
$$

Here we use notations $\triangle_{g}$ and $\nabla_{g}$ to denote the Laplacian and gradient operators under the metric $g$. Note that $u$ and therefore $l$ depends on the boundary value $\left.u\right|_{\partial \Omega}$ as well as the metric $g$. Consider the restriction of the tensor defined in (1) on the gradient vector field $l$ defined in (2) and let $F$ be the resulting 1-form. We then obtain the following inhomogeneous equation related to the conformal Killing field:

$$
l\rfloor\left(\mathcal{L}_{X}(g)-\left(e^{\sigma} \nabla \cdot\left(e^{-\sigma} X\right)\right) g\right)=F .
$$

Given a metric $g$, the inverse problem considered in this paper asks whether one can obtain information about $X$ and $l$ from knowledge of $F$, assuming $\left.X\right|_{\partial \Omega}=0$. In this paper we prove that both inner products $\langle l, X\rangle_{g}$ and $\left\langle l^{\perp}, X\right\rangle_{g}$ are uniquely determined by $F$, where $l^{\perp}$ stands for the unique vector perpendicular to $l$ with $\left\|l^{\perp}\right\|=\|l\|$ in the counter-clockwise direction under the metric $g$.

Theorem 1. Let $g$ be a $C^{2, \alpha}$ Riemannian metric on $\bar{\Omega}$. Let $X$ be a $C^{2, \alpha}$ vector field on $\bar{\Omega}$ satisfying $\left.X\right|_{\partial \Omega}=0$, and $l$ a $C^{2, \alpha}$ gradient field satisfying equation (2). Let $F$ be a $C^{1, \alpha} 1$-form on $\bar{\Omega}$ such that

$$
l\rfloor\left(\mathcal{L}_{X}(g)-\left(e^{\sigma} \nabla \cdot\left(e^{-\sigma} X\right)\right) g\right)=F .
$$

Then both $\langle l, X\rangle_{g}$ and $\left\langle l^{\perp}, X\right\rangle_{g}$ are uniquely determined by $F$.

The main feature of Theorem 1 is that both $X$ and $l$ are unknown. As one will see later, the assumption that $\left.X\right|_{\partial \Omega}=0$ is crucial in Theorem 1. This assumption guarantees that both $\langle l, X\rangle_{g}$ and $\left\langle l^{\perp}, X\right\rangle_{g}$ can be determined by $F$ alone. In fact, the assumption $\left.X\right|_{\partial \Omega}=0$ guarantees $\left.\langle l, X\rangle_{g}\right|_{\partial \Omega}=0$ and $\left.\left\langle l^{\perp}, X\right\rangle_{g}\right|_{\partial \Omega}=0$ for any $l$, and thus eliminates the need of any addtional information about $l$ at the boundary. If we consider the case where $l$ is a known gradient field and search for information of $X$ only, then the assumption $\left.X\right|_{\partial \Omega}=0$ is not necessary, as one can see from the following theorem. 
Theorem 2. Let $g$ be a $C^{2, \alpha}$ Riemannian metric on $\bar{\Omega}$. Let $X$ be a $C^{2, \alpha}$ vector field on $\bar{\Omega}$ and $l$ a $C^{2, \alpha}$ gradient field satisfying equation (2). Let $F$ be a $C^{1, \alpha} 1$-form on $\bar{\Omega}$, such that

$$
l\rfloor\left(\mathcal{L}_{X}(g)-\left(e^{\sigma} \nabla \cdot\left(e^{-\sigma} X\right)\right) g\right)=F .
$$

Then both $\langle l, X\rangle_{g}$ and $\left\langle l^{\perp}, X\right\rangle_{g}$ are uniquely determined by $F,\left.X\right|_{\partial \Omega}$ and $\left.l\right|_{\partial \Omega}$.

A consequence of Theorem 2 is that, when $\left.l\right|_{\partial \Omega}$ is known and $\left.l\right|_{\partial \Omega} \neq 0$, the vector field $X$ can be determined by $F,\left.X\right|_{\partial \Omega}$ and $\left.l\right|_{\partial \Omega}$. This is due to the fact that any nonconstant solution to a two-dimensional elliptic equation carries only discrete critical points [B] and thus $X$ is determined by the inner products $\langle l, X\rangle_{g}$ and $\left\langle l^{\perp}, X\right\rangle_{g}$. Note that in Theorem 1 , the vector field $X$ cannot be determined by $F$ alone since the vector field $l$ is also unknown. If in Theorem 1 we assume $F=0$, then both $\langle l, X\rangle_{g}$ and $\left\langle l^{\perp}, X\right\rangle_{g}$ must equal zero, as one shall see from the proof of Theorem 1.

Theorem 3. Let $g$ be a $C^{2, \alpha}$ Riemannian metric on $\bar{\Omega}$. Let $X$ be a $C^{2, \alpha}$ vector field on $\bar{\Omega}$ satisfying $\left.X\right|_{\partial \Omega}=0$, and $l$ a $C^{2, \alpha}$ gradient field satisfying equation (2). If

$$
l\rfloor\left(\mathcal{L}_{X}(g)-\left(e^{\sigma} \nabla \cdot\left(e^{-\sigma} X\right)\right) g\right) l=0
$$

in $\Omega$, then both $\langle l, X\rangle_{g}$ and $\left\langle l^{\perp}, X\right\rangle_{g}$ are equal to zero in $\Omega$.

If one chooses $\left.l\right|_{\partial \Omega} \neq 0$ in Theorem 3, then we can conclude $X=0$ in $\Omega$. Theorem 3 sharpens, in the setting considered in this paper, some of the classical theorems regarding conformal Killing field obtained in [Y].

\section{Proof of the theorems}

We assume readers are familiar with some basic concepts of Cartan's moving frame method on Riemannian manifolds.

For any point in $\Omega$ there exists an open neighborhood $U$ of the point in which one can construct two unit vector fields $e_{1}$ and $e_{2}$ such that the pair $\left\{e_{1}, e_{2}\right\}$ forms an orthonormal frame (under the metric $g$ ). Let $\omega_{1}$ and $\omega_{2}$ be two 1-forms on $U$ such that the pair $\left\{\omega_{1}, \omega_{2}\right\}$ forms the dual frame of $\left\{e_{1}, e_{2}\right\}$. We can write

$$
F=F_{1} \omega_{1}+F_{2} \omega_{2}
$$

with two components $F_{1}$ and $F_{2}$ defined on $U$. Then the equation in Theorem 1 can be rewritten as two equations

$$
\left(\mathcal{L}_{X} g-e^{\sigma} \nabla_{g} \cdot\left(e^{-\sigma} X\right) g\right)\left(l, e_{k}\right)=F_{k}, \quad k=1,2,
$$

in which the tensor $\mathcal{L}_{X} g-e^{\sigma} \nabla_{g} \cdot\left(e^{-\sigma} X\right) g$ is applied to two vector fields $l$ and $e_{1}$ or $e_{2}$ and the vector field $l$ satisfies equation (2).

From the definition of the Lie derivative $[\mathrm{He}]$ and the following simple relation

$$
e^{\sigma} \nabla_{g} \cdot\left(e^{-\sigma} X\right)=\left\langle\nabla_{g} \sigma, X\right\rangle_{g}-\nabla_{g} \cdot X,
$$

one can further rewrite equation (4) in the following form:

$$
\begin{aligned}
X\left\langle l, e_{k}\right\rangle_{g} & -\left\langle[X, l], e_{k}\right\rangle_{g}-\left\langle l,\left[X, e_{k}\right]\right\rangle_{g}+\left\langle\nabla_{g} \sigma, X\right\rangle_{g}\left\langle l, e_{k}\right\rangle_{g} \\
& -\left(\nabla_{g} \cdot X\right)\left\langle l, e_{k}\right\rangle_{g}=F_{k}, \quad k=1,2 .
\end{aligned}
$$


Here and in the rest of the paper, we use $X f$ to denote the application of the vector field $X$ on the function $f$, which is the directional derivative of $f$ under the vector field $X$, and use $[X, Y]$ to denote the Lie bracket of vectors $X$ and $Y$.

Let us denote by $\mathcal{D}$ the covariant differentiation associated to the metric $g$ and by $\mathcal{D}_{Y}$ the covariant differentiation in the direction of a vector field $Y$. Then

$$
[X, Y]=\mathcal{D}_{X} Y-\mathcal{D}_{Y} X .
$$

$\mathcal{D}$ is best characterized through the connection forms $\omega_{i j}, i, j=1,2$, in the following equations:

$$
\mathcal{D} e_{k}=\sum_{i=1}^{2} \omega_{k i} e_{i}, \quad k=1,2,
$$

with

$$
\omega_{i j}+\omega_{j i}=0, \quad i, j=1,2 .
$$

Using the connection forms one can express the covariant derivative of a vector field $v=v_{1} e_{1}+v_{2} e_{2}$ by the following formula:

$$
\mathcal{D} v=\sum_{i=1}^{2}\left(d v_{i}\right) e_{i}+\sum_{i, j=1}^{2} v_{j} \omega_{j i} e_{i}=\sum_{i, j=1}^{2} v_{i, j} \omega_{j} e_{i},
$$

where $d$ stands for the exterior differentiation and the function $v_{i, j}, i, j=1,2$, are the components of the covariant derivative. Similarly, the first and the second order covariant derivatives of a scalar function $f$, when expressed by $f_{i}$ and $f_{i j}, i, j=1,2$, are given by the following formulas:

$$
\begin{gathered}
d f=\sum_{i=1}^{2} f_{i} \omega_{i}, \\
\sum_{j=1}^{2} f_{i j} \omega_{j}=d f_{i}+\sum_{j=1}^{2} f_{j} \omega_{j i}, \quad i=1,2 .
\end{gathered}
$$

Note that in this case $f_{i j}$ is symmetric in $i$ and $j$.

Under the above setting we can express the following differential operators in terms of the covariant derivatives:

$$
\begin{gathered}
\nabla_{g} f=f_{1} e_{1}+f_{2} e_{2}, \\
\triangle_{g} f=f_{11}+f_{22}, \\
\nabla_{g} \cdot F=F_{1,1}+F_{2,2} .
\end{gathered}
$$

We now calculate the left-hand side of (5). Under the orthonormal frame $\left\{e_{1}, e_{2}\right\}$ one can write

$$
l=l_{1} e_{1}+l_{2} e_{2}, \quad X=X_{1} e_{1}+X_{2} e_{2} .
$$


Then

$$
X\left\langle l, e_{k}\right\rangle_{g}=\sum_{j=1}^{2} X_{j} e_{j} l_{k}=\sum_{j=1}^{2} X_{j}\left(d l_{k}\right)\left(e_{j}\right)
$$

where $\left(d l_{k}\right)\left(e_{j}\right)$ means the exterior derivative of $l_{k}$ in the direction of $e_{j}$. By using (6), (7), (9) and the definition of the Lie bracket,

$$
\begin{aligned}
{\left[X, e_{k}\right] } & =\mathcal{D}_{X} e_{k}-\mathcal{D}_{e_{k}} X \\
& =\sum_{j=1}^{2}\left(X_{j} \mathcal{D}_{e_{j}} e_{k}-\mathcal{D}_{e_{k}}\left(X_{j} e_{j}\right)\right) \\
& =\sum_{i, j=1}^{2} X_{j} \omega_{k i}\left(e_{j}\right) e_{i}-\sum_{j=1}^{2} d X_{j}\left(e_{k}\right) e_{j}-\sum_{i, j=1}^{2} X_{j} \omega_{j i}\left(e_{k}\right) e_{i},
\end{aligned}
$$

so we have (by (8))

$$
\begin{aligned}
X & \left\langle l, e_{k}\right\rangle_{g}-\left\langle l,\left[X, e_{k}\right]\right\rangle_{g} \\
& =\sum_{j=1}^{2} X_{j}\left(d l_{k}\right)\left(e_{j}\right)-\sum_{i, j=1}^{2} X_{j} l_{i} \omega_{k i}\left(e_{j}\right)+\sum_{j=1}^{2} l_{j} d X_{j}\left(e_{k}\right)+\sum_{i, j=1}^{2} X_{j} l_{i} \omega_{j i}\left(e_{k}\right) \\
& =\sum_{j=1}^{2} X_{j}\left(d l_{k}\right)\left(e_{j}\right)+\sum_{i, j=1}^{2} X_{j} l_{i} \omega_{i k}\left(e_{j}\right)+\sum_{j=1}^{2} l_{j} d X_{j}\left(e_{k}\right)+\sum_{i, j=1}^{2} l_{j} X_{i} \omega_{i j}\left(e_{k}\right) \\
& =\sum_{j=1}^{2}\left(X_{j} l_{k, j}+l_{j} X_{j, k}\right) .
\end{aligned}
$$

By (6), (7) again,

$$
\begin{aligned}
{[X, l]=} & \mathcal{D}_{X} l-\mathcal{D}_{l} X \\
= & \sum_{i, j=1}^{2}\left(X_{i} \mathcal{D}_{e_{i}}\left(l_{j} e_{j}\right)-l_{j} \mathcal{D}_{e_{j}}\left(X_{i} e_{i}\right)\right) \\
= & \sum_{i, j=1}^{2}\left(X_{i}\left(d l_{j}\right)\left(e_{i}\right) e_{j}+X_{i} l_{j} \mathcal{D}_{e_{i}} e_{j}-l_{j} d X_{i}\left(e_{j}\right) e_{i}-X_{i} l_{j} \mathcal{D}_{e_{j}} e_{i}\right) \\
= & \sum_{i, j=1}^{2} X_{i}\left(d l_{j}\right)\left(e_{i}\right) e_{j}+\sum_{i, j, m=1}^{2} X_{i} l_{j} \omega_{j m}\left(e_{i}\right) e_{m} \\
& -\sum_{i, j=1}^{2} l_{j} d X_{i}\left(e_{j}\right) e_{i}-\sum_{i, j, m=1}^{2} X_{i} l_{j} \omega_{i m}\left(e_{j}\right) e_{m},
\end{aligned}
$$


so we have (by (9))

$$
\begin{aligned}
\left\langle[X, l], e_{k}\right\rangle_{g}= & \sum_{i, j=1}^{2}\left(X_{i}\left(d l_{k}\right)\left(e_{i}\right)+X_{i} l_{j} \omega_{j k}\left(e_{i}\right)\right) \\
& -\sum_{j=1}^{2} l_{j} d X_{k}\left(e_{j}\right)-\sum_{i, j=1}^{2} X_{i} l_{j} \omega_{i k}\left(e_{j}\right) \\
= & \sum_{i, j=1}^{2}\left(X_{i}\left(d l_{k}+l_{j} \omega_{j k}\right)\left(e_{i}\right)-l_{i}\left(d X_{k}+X_{j} w_{j k}\right)\left(e_{i}\right)\right) \\
= & \sum_{i=1}^{2}\left(X_{i} l_{k, i}-l_{i} X_{k, i}\right) .
\end{aligned}
$$

From (12) and (14) we have

$$
\begin{array}{r}
\left\langle\nabla_{g} \sigma, X\right\rangle_{g}\left\langle l, e_{k}\right\rangle_{g}-\left(\nabla_{g} \cdot X\right)\left\langle l, e_{k}\right\rangle_{g} \\
=\sum_{j=1}^{2} \sigma_{j} X_{j} l_{k}-\left(X_{1,1}+X_{2,2}\right) l_{k} .
\end{array}
$$

Combining (15)-(17) together we have that the left-hand side of (5) can be expressed as

$$
\sum_{j=1}^{2}\left(l_{j} X_{k, j}+l_{j} X_{j, k}+\sigma_{j} X_{j} l_{k}\right)-\left(X_{1,1}+X_{2,2}\right) l_{k}, k=1,2 .
$$

When $k=1$, (18) can be expressed as

$$
l_{1} X_{1,1}+l_{2} X_{2,1}-\left(-l_{2} X_{1,2}+l_{1} X_{2,2}\right)+\left(\sigma_{1} X_{1}+\sigma_{2} X_{2}\right) l_{1},
$$

and therefore the first equation of (5), i.e. the case with $k=1$, can be expressed as follows:

$$
\left\langle l, \mathcal{D}_{e_{1}} X\right\rangle_{g}-\left\langle l^{\perp}, \mathcal{D}_{e_{2}} X\right\rangle_{g}+\left(\sigma_{1} X_{1}+\sigma_{2} X_{2}\right) l_{1}=F_{1} .
$$

Here we have used the simple fact that

$$
l^{\perp}=-l_{2} e_{1}+l_{1} e_{2} .
$$

Similarly, when $k=2$, (18) can be simplified to

$$
l_{1} X_{2,1}+l_{2} X_{2,2}+\left(-l_{2} X_{1,1}+l_{1} X_{2,1}\right)+\left(\sigma_{1} X_{1}+\sigma_{2} X_{2}\right) l_{2},
$$

and the second equation of (5), i.e. the case with $k=2$, can be rewritten as

$$
\left\langle l, \mathcal{D}_{e_{2}} X\right\rangle_{g}-\left\langle l^{\perp}, \mathcal{D}_{e_{1}} X\right\rangle_{g}+\left(\sigma_{1} X_{1}+\sigma_{2} X_{2}\right) l_{2}=F_{2} .
$$

Set

$$
p=\langle l, X\rangle_{g}, \quad q=\left\langle l^{\perp}, X\right\rangle_{g} .
$$

Then

$$
\begin{gathered}
p_{k}=e_{k} p=\left\langle\mathcal{D}_{e_{k}} l, X\right\rangle_{g}+\left\langle l, \mathcal{D}_{e_{k}} X\right\rangle_{g}, \\
q_{k}=e_{k} q=\left\langle\mathcal{D}_{e_{k}} l^{\perp}, X\right\rangle_{g}+\left\langle l^{\perp}, \mathcal{D}_{e_{k}} X\right\rangle_{g} .
\end{gathered}
$$


Clearly, with $p$ and $q$ we can rewrite equations (19) and (20) in the forms

$$
\begin{gathered}
p_{1}-q_{2}-\left\langle\mathcal{D}_{e_{1}} l, X\right\rangle_{g}+\left\langle\mathcal{D}_{e_{2}} l^{\perp}, X\right\rangle_{g}+\left(\sigma_{1} X_{1}+\sigma_{2} X_{2}\right) l_{1}=F_{1}, \\
p_{2}+q_{1}-\left\langle\mathcal{D}_{e_{2}} l, X\right\rangle_{g}-\left\langle\mathcal{D}_{e_{1}} l^{\perp}, X\right\rangle_{g}+\left(\sigma_{1} X_{1}+\sigma_{2} X_{2}\right) l_{2}=F_{2} .
\end{gathered}
$$

Since $l_{k}, k=1,2$, are covariant derivatives of the solution of the equation

$$
\triangle_{g} u-\left\langle\nabla_{g} \sigma, \nabla_{g} u\right\rangle_{g}=0,
$$

we have that $l_{i, j}, i, j=1,2$, are the second order covariant derivatives of the solution $u$, and therefore

$$
l_{i, j}=u_{i j}=u_{j i}=l_{j, i}, i, j=1,2 .
$$

Using this fact as well as equation (2), we have

$$
\begin{gathered}
-\left\langle\mathcal{D}_{e_{1}} l, X\right\rangle_{g}+\left\langle\mathcal{D}_{e_{2}} l^{\perp}, X\right\rangle_{g}+\left(\sigma_{1} X_{1}+\sigma_{2} X_{2}\right) l_{1} \\
=-l_{1,1} X_{1}-l_{2,2} X_{1}+\left(\sigma_{1} X_{1}+\sigma_{2} X_{2}\right) l_{1} \\
=-\left(\nabla_{g} \cdot l\right) X_{1}+\left(\sigma_{1} X_{1}+\sigma_{2} X_{2}\right) l_{1} \\
=-\left\langle\nabla_{g} \sigma, l\right\rangle_{g} X_{1}+\left(\sigma_{1} X_{1}+\sigma_{2} X_{2}\right) l_{1} \\
=-\sigma_{2} l_{2} X_{1}+\sigma_{2} l_{1} X_{2}=\sigma_{2} q .
\end{gathered}
$$

Similarly,

$$
\begin{gathered}
-\left\langle\mathcal{D}_{e_{2}} l, X\right\rangle_{g}-\left\langle\mathcal{D}_{e_{1}} l^{\perp}, X\right\rangle_{g}+\left(\sigma_{1} X_{1}+\sigma_{2} X_{2}\right) l_{2} \\
=-l_{2,2} X_{2}-l_{1,1} X_{2}+\left(\sigma_{1} X_{1}+\sigma_{2} X_{2}\right) l_{2} \\
=-\left(\nabla_{g} \cdot l\right) X_{2}+\left(\sigma_{1} X_{1}+\sigma_{2} X_{2}\right) l_{2} \\
=-\left\langle\nabla_{g} \sigma, l\right\rangle_{g} X_{2}+\left(\sigma_{1} X_{1}+\sigma_{2} X_{2}\right) l_{2} \\
=-\sigma_{1} l_{1} X_{2}+\sigma_{1} l_{2} X_{1}=-\sigma_{1} q .
\end{gathered}
$$

Hence, (21) and (22) become

$$
\begin{aligned}
& p_{1}-q_{2}+\sigma_{2} q=F_{1}, \\
& p_{2}+q_{1}-\sigma_{1} q=F_{2} .
\end{aligned}
$$

Taking covariant derivatives on both sides of (23) and (24) and then substracting the two equations yield

$$
q_{11}+q_{22}-\left(\sigma_{1} q\right)_{1}-\left(\sigma_{2} q\right)_{2}=F_{2,1}-F_{1,2},
$$

i.e.

$$
\triangle_{g} q-\nabla_{g} \cdot(\sigma q)=-\nabla_{g} \cdot F^{\perp} .
$$

Clearly, equation (25) holds in the entire domain $\Omega$. By the uniqueness of solutions to elliptic equations we have that $q$ is uniquely determined by $F$ and $\left.q\right|_{\partial \Omega}$. In the case of Theorem 1, we have $\left.X\right|_{\partial \Omega}=0$ which implies $\left.q\right|_{\partial \Omega}=0$, and therefore $q$ is uniquely determined by $F$ alone. In the general case as in Theorem $2,\left.q\right|_{\partial \Omega}$ is determined by both $\left.l\right|_{\partial \Omega}$ and $\left.X\right|_{\partial \Omega}$ and therefore $q$ is determined by $F,\left.l\right|_{\partial \Omega}$ and $\left.X\right|_{\partial \Omega}$. Clearly, if both $F=0$ and $\left.X\right|_{\partial \Omega}=0$ are assumed, then $q=0$, as claimed by Theorem 3 .

On the other hand, one can derive a similar equation for $p$ :

$$
\triangle_{g} p+\left(\sigma_{2} q\right)_{1}-\left(\sigma_{1} q\right)_{2}=F_{1,1}+F_{2,2},
$$

and one can get the same results for $p$ as we did for $q$. This completes the proof. 


\section{REFERENCES}

[B] L. Bers, F. John, and M. Schechter, Partial differential equations, Interscience Publishers, New York, 1964. reprint MR 82c:35001

[H-Su] D. Hervas and Z. Sun, An inverse boundary value problem for quasilinear elliptic equations, to appear in Comm. in PDE.

[He] R. Hermann, Differential geometry and the calculus of variations, Academic Press, New York and London, 1968. MR 38:1635

[I 1] V. Isakov, On uniqueness in inverse problems for semilinear parabolic equations, Arch. Rat. Mech.Anal. 124 (1993), 1-12.

[I 2] V. Isakov, Uniqueness of recovery of some systems of semilinear partial differential equations, Inverse Problems 17 (2001) 607-618. MR 2002g:35213

[I-N] V. Isakov and A. Nachman, Global uniqueness for a two-dimensional semilinear elliptic inverse problem, Trans. of AMS 347 (1995), 3375-3390. MR 95m:35202

[I-S] V. Isakov and J. Sylvester, Global uniqueness for a semilinear elliptic inverse problem, Comm. Pure Appl. Math. 47 (1994), 1403-1410. MR 95h:35243

[Su] Z. Sun, On a quasilinear inverse boundary value problem, Math. Z. 221 (1996), 293-305. MR 96m:35109

[Su-U] Z. Sun and G. Uhlmann, Inverse problems in quasilinear anisotropic media, Amer. J. of Math. 119 (1997), 771-797. MR 98g:35216

[U] G. Uhlmann, Developments in inverse problems since Calderon's foundational paper, Harmonic Analysis and Pde, University of Chicago Press, 1999. MR 2000m:35181

[Y] K. Yano, Integral formulas in Riemannian Geometry, Marcel Dekker, Inc., New York, 1970. MR 44:2174

Department of Mathematics, Wichita State University, Wichita, Kansas 67260-0033

E-mail address: ziqi.sun@wichita.edu 